\title{
AVALIAÇÃO DOS ÍNDICES HEMATIMÉRICOS EM IDOSOS CORRELACIONANDO ANEMIAS NA CIDADE DE BREJO SANTO - CE
}

\author{
EVALUATION OF HEMATIMERIC INDEXES IN THE ELDERLY \\ CORRELATING ANEMIA IN THE CITY OF BREJO SANTO - CE
}

Cícero Lasaro Gomes Moreira ${ }^{1}$

Alexsandra Laurindo Leite ${ }^{2}$

Francisco Eduardo Ferreira Alves ${ }^{3}$

RESUMO: Objetivo: Tendo em vista as considerações feitas no estudo, a relevância e o objetivo deste foram avaliar a prevalência de anemias correlacionando ao diagnóstico diferencial através da avaliação dos índices hematiméricos de um grupo de idosos com idade a partir de 60 anos da cidade de Brejo Santo, Ceará, no ano de 2016. Metodologia: Trata-se de uma pesquisa de campo, descritiva e exploratória com abordagem quantitativa realizada em um laboratório de análises clínicas de um hospital da rede pública na cidade de Brejo Santo. Esta foi realizada através da análise dos hemogramas dos indivíduos participantes, avaliando-se os valores de Eritrócitos, Hemoglobina, Hematócrito, bem como os índices hematiméricos, sendo estes o Volume Corpuscular Médio (VCM), Hemoglobina Corpuscular Média (HCM) e a Concentração de Hemoglobina Corpuscular Média (CHCM), bem como os resultados dos questionários respondidos no ato da realização do exame. Os valores de referência utilizados para a avaliação do hemograma foram baseados nos valores emitidos pelo laboratório onde foi realizada a pesquisa. A compilação estatística dos dados foi realizada usando o programa SPSS Statistics, versão 23. Resultados: A faixa etária dos indivíduos participantes variou de 60 a 97 anos com média de 72,30 anos, destes, $65 \%$ eram do sexo masculino e $35 \%$ eram do sexo feminino. Os resultados obtidos mostraram que, a partir da análise dos hemogramas dos participantes, $25 \%$ apresentaram algum tipo de anemia, sendo que $15 \%$ eram do sexo masculino e $10 \%$ do sexo feminino. Além disso, $7,50 \%$ apresentaram anemia microcítica e hipocrômica, e 17,50\%, anemia normocítica e normocrômica. Nos indivíduos sugestivos de anemia, 15\% apresentaram anisocitose. Para os indivíduos não-anêmicos, 22,50\% apresentaram anisocitose. Conclusão: A partir da avaliação

\footnotetext{
1 Biomédico especialista em Hematologia Clínica - AUTOR.

2 Docente do Curso de Biomedicina da Faculdade Santa Maria.

3 Biomédico. Pós- Graduado em Hematologia Clinica-UNILEAO. Pós-Graduando em Microbiologia clínica- UNILEAO. Mestrando em Ciências e Tecnologia em Saúde- UEPB.
} 
dos dados referidos no presente estudo, pode-se concluir que, de acordo com a amostragem avaliada, a prevalência de anemias na população idosa ainda é preocupante, já que um quarto destes se encontrava com quadros anêmicos.

Palavras chave: Anemia. Hemoglobinopatias. Idosos. Índices hematimétricos.

ABSTRACT: Objective: In view of the considerations made in this study, its relevance and objective were to evaluate the prevalence of anemia correlated to the differential diagnosis through the evaluation of hematimeric indexes of a group of elderly individuals aged 60 years or more from the city of Brejo Santo, Ceará, in the year 2016. Methodology: This is a field, exploratory and descriptive research, with quantitative approach, performed in a laboratory of a public hospital in the city of Brejo Santo. The participants' blood tests were analyzed, assessing the values of Erythrocytes, Hemoglobin, Hematocrit, as well as hematimeric indexes: Mean Corpuscular Volume (MCV), Mean Corpuscular Hemoglobin $(\mathrm{MCH})$, and Mean Corpuscular Hemoglobin Concentration (MCHC), as well as the results of the questionnaires answered at the collection. The reference values used for the hemogram evaluation were based on the values emitted by the laboratory where the research was carried out. Data statistical compilation was done using the program SPSS Satistics, version 23. Results: The age of the studied group ranged from 60 to 97 years with a mean of 72.30 years, of these, $65 \%$ were male and $35 \%$ female. The results showed that, from the analysis of the participants' blood tests, $25 \%$ had some type of anemia, $15 \%$ male and $10 \%$ female. In addition, $7.50 \%$ had microcytic and hypochromic anemia, and $17.50 \%$, normocytic and normochromic anemia. In individuals suggestive of anemia, $15 \%$ had anisocytosis. For non-anemic individuals, $22.50 \%$ had anisocytosis. Conclusion: The data evaluation revealed that, in the present study, according to the evaluated sample, the prevalence of anemias in the elderly population is still worrying, since a quarter of these had anemic frames.

Keywords: Anemia. Hemoglobinopathies. Elderly. Hematimeric indexes. 


\section{INTRODUÇÃO}

O envelhecimento é uma progressão que abrange todo o mundo e que pode ser observado devido à ocorrência do aumento da expectativa de vida, diminuição dos índices de natalidade, bem como da fecundidade. Devido a isso, observa-se, no Brasil e em países em desenvolvimento, que o envelhecimento da população vem se mostrando vertiginoso desde os anos de 1960 (MILAGRES et al., 2014). Além disso, o envelhecimento vem se tornando um assunto bastante debatido devido à maior longevidade dos indivíduos. Logo, o tamanho da pirâmide populacional vem se acidentando à medida que o tempo passa por causa do acréscimo da população idosa em relação à população total, bem como por adiantamentos na medicina e progresso da qualidade de vida (BUFFON et al., 2015).

Por esses motivos, os idosos encontram-se mais suscetíveis a desencadear quadros anêmicos, principalmente do tipo carencial, como aqueles por deficiência de ferro, vitamina B12 e ácido fólico, por estarem relacionados com a má nutrição e o avançar da idade, dentre vários outros fatores (NEKEL, 2013).

Por ser uma das condições que se encontram presentes em várias patologias, a anemia é definida, segundo o Guia de Evidências do Ministério da Saúde, como uma diminuição nos níveis séricos de hemoglobina circulante na corrente sanguínea, evidenciada por uma diminuição, tanto qualitativa como quantitativa, do número de eritrócitos, sendo de grande importância o seu diagnóstico (BRASIL 2015).

Apesar de a diminuição dos níveis séricos de hemoglobina estarem relacionados com o envelhecimento, é perceptível que, segundo a Organização Mundial da Saúde (OMS), mais de 10\% dos indivíduos com idade superior a 60 anos apresentam casos de anemias, onde a anemia ferropriva enquadra-se como uma das principais do tipo carencial, acometendo a população de idosos, sendo que esses dados só tendem a aumentar com o avançar da idade (GUALANDRO et al., 2010). 
A avaliação dos índices hematiméricos é de grande importância para que seja possível o diagnóstico diferencial das anemias, pois, do ponto de vista clínico, as mesmas apresentam causas, tratamentos e prognósticos diferenciados. $O$ diagnóstico diferencial pode ser feito através da avaliação do metabolismo do ferro, dosagem de Hemoglobina Glicosilada $\left(\mathrm{HbA}_{2}\right)$ e da eletroforese de hemoglobina (MATOS et al., 2012).

Assim, é importante manter os valores de referência do CHCM com critérios rígidos, pois, com esse resultado, consegue-se liberar laudos com alta confiabilidade diretamente dos aparelhos de automação em hematologia, fazendo com que o clínico possa ter mais segurança no diagnóstico e acompanhamento de seus pacientes (BRUM e FRIZZO, 2013).

Com o objetivo de aperfeiçoar o diagnóstico complementar dessas anemias, foram utilizados os resultados dos parâmetros hematológicos obtidos através do hemograma, como o Volume Corpuscular Médio (VCM) e a Hemoglobina Corpuscular Média (HCM), dentre outros.

Dessa forma, este estudo objetivou avaliar os índices hematiméricos de um grupo de indivíduos com idade igual ou acima de 60 anos da cidade de Brejo Santo $\mathrm{CE}$, correlacionando ao diagnóstico diferencial e à prevalência de anemias.

\section{MATERIAIS E MÉTODOS}

Trata-se de uma pesquisa de campo, descritiva e exploratória, aprovada pelo Comitê de Ética em Pesquisa da Faculdade Santa Maria (parecer 1.741.369), cujo principal interesse foi avaliar a prevalência de anemias, correlacionando ao diagnóstico diferencial através da avaliação dos índices hematiméricos de 40 indivíduos com idade a partir de 60 anos. Este número justifica-se pela quantitativo populacional da cidade de Brejo Santo, que, em 2016, era de 48.451 habitantes e densidade demográfica, em 2010, de 68,12hab/km² (IBGE, 2016). O estudo em questão apresenta abordagem do tipo quantitativa, e foi realizado em um laboratório de análises clínicas de um hospital da rede pública na cidade de Brejo Santo - CE. 
Os dados das amostras de sangue colhidas foram analisados no laboratório de análises clinicas, onde, ao serem coletadas em tubo de coleta contendo EDTA, a amostra de sangue total foi analisada por meio do ADVIA 120, equipamento automatizado que forneceu os resultados posteriormente analisados.

Além dos valores emitidos pelo equipamento automatizado, foi realizada uma revisão de lâmina, avaliando a morfologia, coloração e tamanho das células sanguíneas, sendo estas os eritrócitos, leucócitos e plaquetas, comparando se houve discrepância com os resultados emitidos pelo equipamento automatizado.

Para a confecção da lâmina hematológica ou esfregaço sanguíneo, utilizou-se uma coloração de panótico rápido com álcool etílico, eosina e azul de metileno para a visualização e diferenciação celular por microscopia.

Os valores de referência utilizados para a análise dos hemogramas foram baseados nos valores emitidos pelo laboratório no qual a pesquisa foi realizada.

A compilação estatística dos dados foi realizada usando o programa SPSS Statistics versão 23 , a partir do qual foram produzidos gráficos e tabelas, e foram verificados através de comparações das variáveis categóricas de interesse com o percentual do valor mediano da prevalência de anemias, bem como a variação destas.

Todas as etapas da pesquisa foram fundamentadas de acordo com a resolução 510/2016 do Conselho Nacional de Saúde (CNS), a qual regulamenta as pesquisas que envolvem seres humanos, inserindo, sob a perspectiva dos indivíduos e da coletividade, as quatro citações básicas da bioética: a autonomia, a não maleficência, a beneficência e a justiça, objetivando, também, certificar os deveres e direitos no tocante à população científica, aos sujeitos da pesquisa e ao estado.

\section{RESULTADOS E DISCUSSÃO}

O processo de envelhecimento, apesar de ser um fenômeno fisiológico, pode desencadear no indivíduo diversas mudanças no seu metabolismo, principalmente em idosos. A anemia é uma dessas consequências que acomete esses indivíduos. 
Foi realizado um estudo com o objetivo de analisar a prevalência de anemia em 40 idosos com idade a partir de 60 anos, revelando uma prevalência considerável de anemias.

A faixa etádia dos indivíduos participantes variou de 60 a 97 anos. com média de 72,30 anos. Destes, $65 \%$ eram do sexo masculino e $35 \%$ eram do sexo feminino. Os resultados obtidos no estudo mostraram que, a partir da análise dos hemogramas dos participantes, $25 \%$ destes apresentaram alterações hematológicas sugestivas de anemias, sem distinção de sexo, como mostra o gráfico 1.

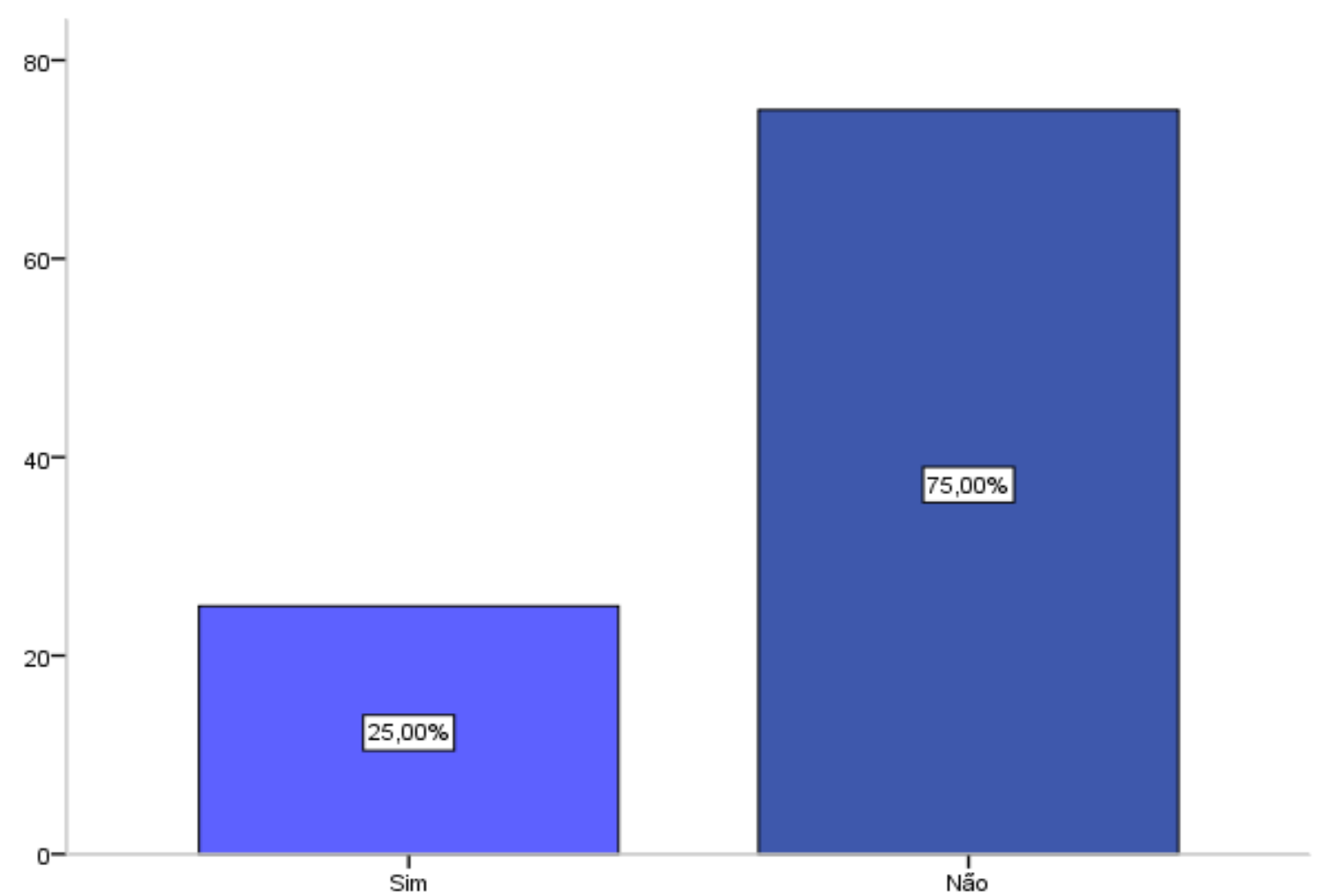

Gráfico 1: Prevalência de alterações hematológicas sem relação com o sexo dos participantes da cidade de Brejo Santo - CE, 2016.

O estudo realizado por Corona; Duarte; Lebrão (2014), que avaliou a prevalência de anemias em indivíduos idosos na cidade de São Paulo - SP, baseado no Estudo SABE (Saúde, Bem-Estar e Envelhecimento), mostrou que, na população estudada, 7,7\% apresentaram anemia. Em contrapartida, o presente estudo apresentou resultados superiores, havendo, dessa forma, uma discordância. Já o estudo de Corrêa et al. (2004), que avaliou a prevalência de anemias em pacientes 
internados na Clínica Médica do Hospital Nossa Senhora da Conceição (HNSC), Tubarão-SC, mostrou resultados semelhantes, com prevalência de $28,3 \%$ de pacientes anêmicos, enquanto que o estudo de Santos (2009), realizado em São Paulo Ageing and Health Study, mostrou prevalência de 10,4\%.

Nesta perspectiva, Rosenfeld (2012) ressalta que um organismo com as contagens normais é capaz de produzir e extinguir as células do sangue em perfeito equilíbrio, sustentando a quantidade e a qualidade das células em circulação. Logo, esse processo evitará infecções e sangramentos, garantindo fornecimento apropriado de oxigênio às células.

A prevalência de alterações hematológicas relacionada com o sexo dos indivíduos idosos obtida neste estudo através da avaliação dos índices hematiméricos revelou que, do sexo masculino, 15\% apresentaram alterações e $50 \%$ com valores dentro da normalidade, ao contrário do sexo feminino, que apresentou $10 \%$ e $25 \%$, respectivamente. Os dados estão exemplificados no gráfico 2.

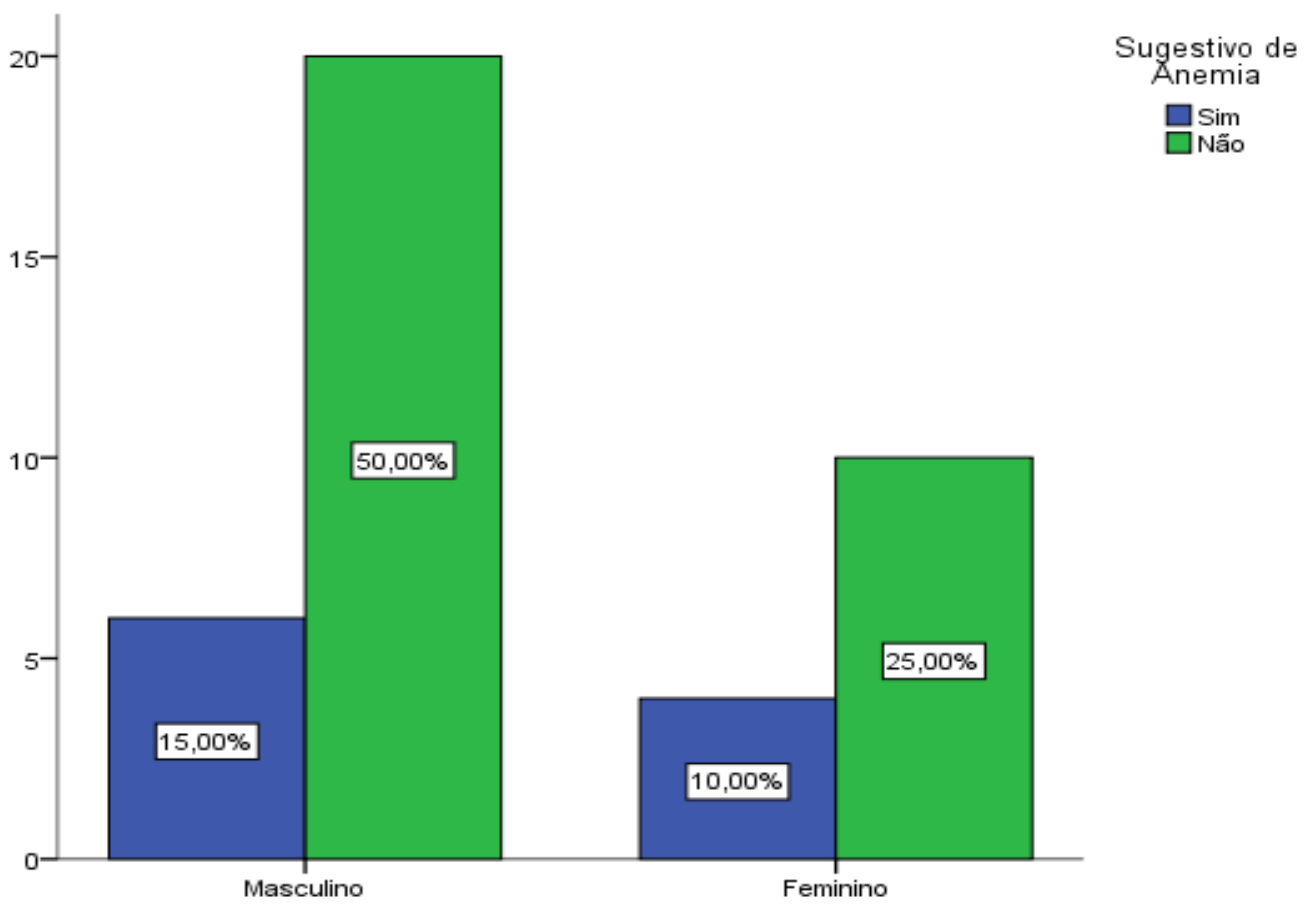

Gráfico 2: Prevalência de alterações hematológicas em idosos de acordo com o sexo na cidade de Brejo Santo - CE, 2016. 
O gráfico 3 exemplifica a prevalência quanto à classificação das alterações hematológicas em pacientes sugestivos de anemias, de acordo com a análise dos índices hematiméricos (VCM, HCM e CHCM), bem como a avaliação do número de eritrócitos, hemoglobina e hematócrito, em que, da população estudada, 7,50\% encontravam-se com anemia microcítica e hipocrômica, sugestivo de deficiência de ferro, e $17,50 \%$ classificados em normocítica e normocrômica, sugestivo de anemias por doenças crônicas ou doenças renais comuns na população.

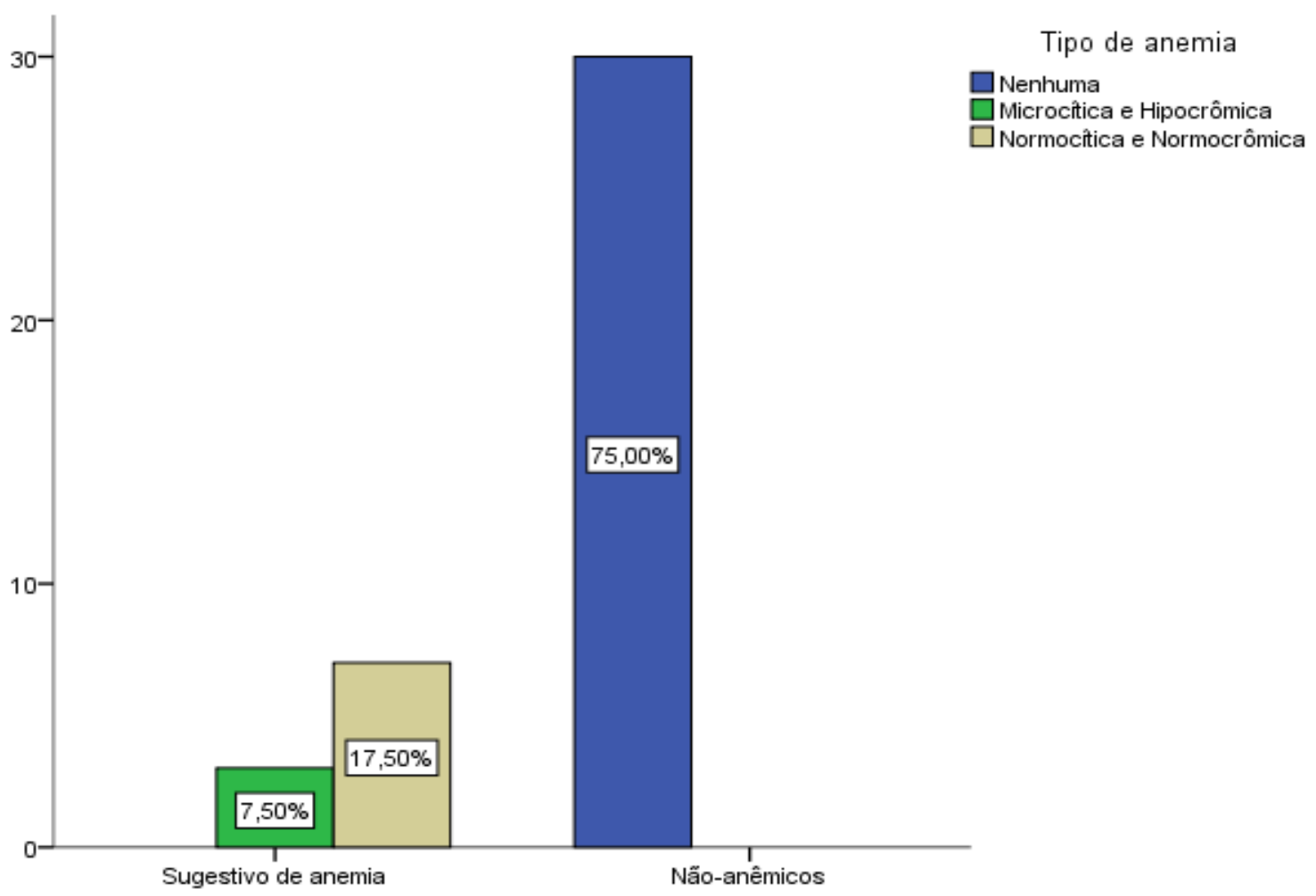

Gráfico 3: Prevalência de alterações hematológicas em pacientes idosos sugestivos de anemia na cidade de Brejo Santo - CE, 2016.

Estudo realizado por Buffon (2014), que analisou a prevalência de anemia em idosos atendidos pela Estratégia Saúde da Família do município de Porto Alegre, mostrou que, dos idosos avaliados, a prevalência de anemia microcítica e hipocrômica foi semelhante, com $6 \%$ e $34 \%$ com anemia do tipo normocítica e normocrômica. 
Levando em consideração os valores encontrados para o RDW (parâmetro que avalia a amplitude de distribuição das hemácias), sendo este um indicador que avalia a presença ou não de anisocitose (termo utilizado para existência de hemácias de tamanhos diferentes numa mesma amostra de sangue), dos 40 indivíduos analisados sugestivos de anemias, $4(10 \%)$ apresentaram valores dentro do padrão de normalidade, enquanto que $6(15 \%)$ apresentaram anisocitose. Dos não-anêmicos, 21 (52,50\%) não apresentaram alterações, e 9 (22,50\%) encontravam-se com RDW alterado, como explanado no gráfico 4.

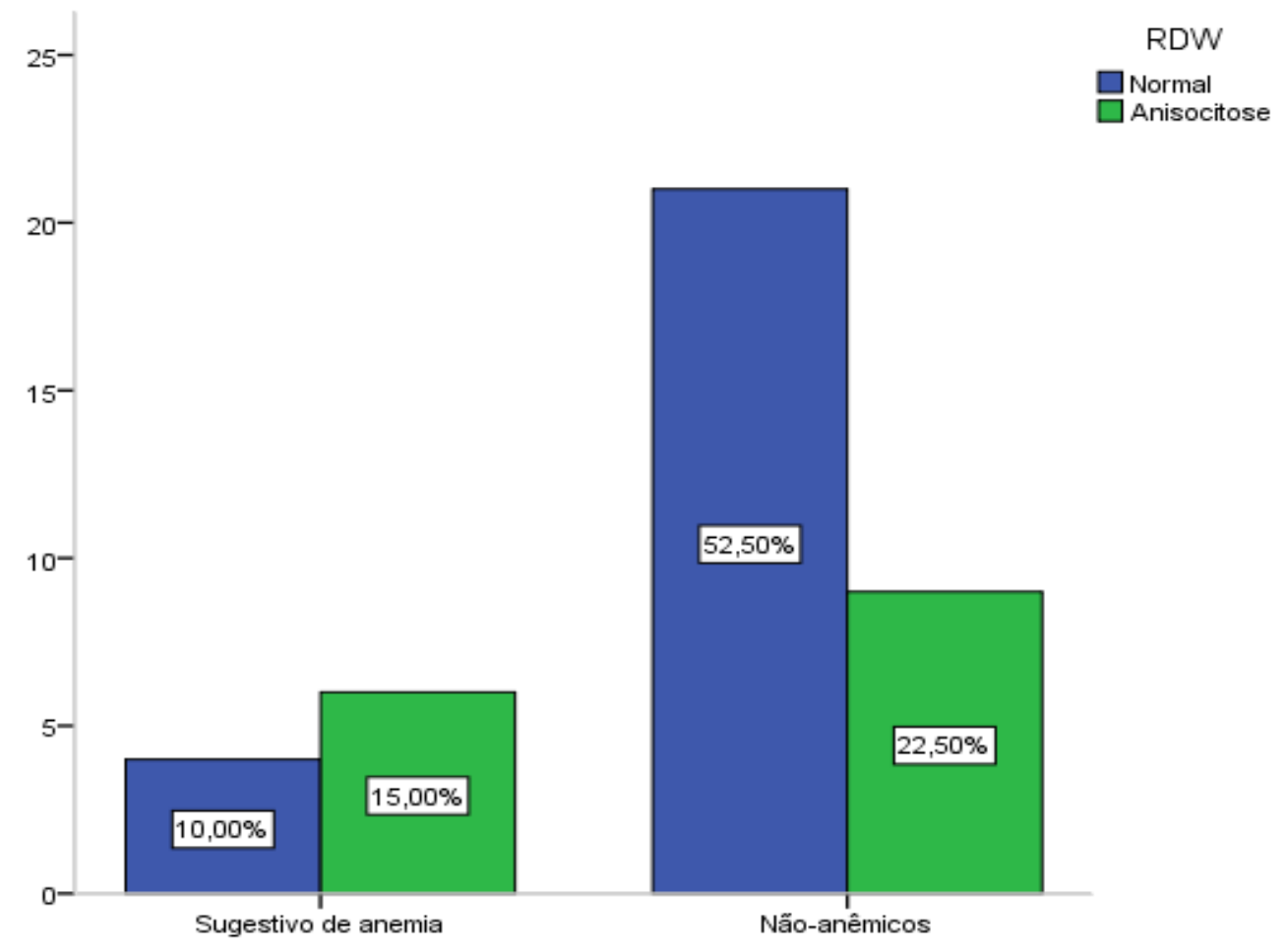

Gráfico 4: RDW - Prevalência de anisocitose sem relação com o sexo dos participantes idosos da cidade de Brejo Santo - CE, 2016.

Os resultados obtidos no estudo de Tomiya, Pinho, \& Cabral (2014), realizado no Hospital das Clínicas da Universidade de Pernambuco, revelaram prevalência de 26,9\% de anisocitose nos participantes idosos anêmicos, discordando do presente estudo, no qual foi encontrado prevalência de 15\%. O estudo de Lopes et al. (2016), realizado no Hospital Universitário Lauro Wanderley, na cidade de João Pessoa - 
PB, mostrou prevalência de anisocitose em $27,4 \%$ dos pacientes, discordando dos resultados obtidos no estudo em questão.

Tais resultados corroboram Cristo et al. (2014), que realizaram uma pesquisa com população constituída por 62 Idosos (acima de 60 anos de idade), e observaram que $19 \%$ dos idosos apresentaram valor de hemoglobina abaixo do que é preconizado pela Organização Mundial de Saúde (OMS), que diz que valores abaixo de $13 \mathrm{~g} / \mathrm{dl}$ para homens e $12 \mathrm{~g} / \mathrm{dl}$ para mulheres caracterizam patologicamente uma anemia. A OMS ainda afirme que é considerada saudável uma população com níveis de anemia nos idosos abaixo de $2,5 \%$ e considera risco de saúde pública valores superiores a $5 \%$.

Machado et al. (2019), com o objetivo de verificar a prevalência de casos de anemia em adultos e idosos brasileiros, obtiveram como resultado a identificação de maiores prevalências de anemias em idosos, pessoas de baixa escolaridade, cor negra, e idosos que residem nos estados do Norte e Nordeste. Ademais, os autores obtiveram que os índices hematimétricos resultaram em 23,2\% VCM reduzido e 10 , $2 \%$ aumentado.

Estudo de Bonjorno et al. (2019) mostrou que, da amostra de 533 hematócritos analisados, 33\% correspondiam a pacientes idosos, sendo $55 \%$ de mulheres e $37 \%$ de homens, estando abaixo do número de referência um total de $41 \%$. Bonjorno et al. (2019) acrescentam que a elevação de resultados hematimétricos alterados, em sua maioria, dá-se pela falha assistencial de saúde a esse público, estando entre as barreiras para a assistência a dificuldade no agendamento de consultas para atendimento médico, assim como carência de programas de acompanhamento de saúde.

Estudo de Sousa (2015), ao analisar resultados de índices hematimétricos de 360 idosos, sendo $67,2 \%$ do sexo feminino, na faixa etária de 60 a 104 anos, mostrou como resultado a prevalência de $12,5 \%$ da amostra como pacientes anêmicos. Além disso, para o autor, fatores como características demográficas, econômicas, sociais, assim como condições cognitivas, estado nutricional e quantidade de doenças crônicas apresentaram associação significativa no diagnóstico da doença. 


\section{CONCLUSÃO}

O hemograma é um exame simples, frequentemente realizado e de suma importância, pois este pode dizer muito sobre a saúde do indivíduo. A avaliação do número de eritrócitos, a hemoglobina e o hematócrito, juntamente com avaliação dos índices hematiméricos (VCM, $\mathrm{HCM}$, e CHCM), pode mostrar alterações eritrocitárias, as quais poderiam dizer se o indivíduo apresenta algum tipo de anemia ou não. Dessa forma, os idosos, por apresentarem mudanças funcionais, biológicas e fisiológicas da velhice, são um grande alvo para o acometimento dessa patologia.

Os resultados apresentados nessa investigação evidenciaram que, da população avaliada, $25 \%$ encontravam-se acometidos por quadros sugestivos de anemia, enquanto que $75 \%$ encontravam-se saudáveis, sendo $15 \%$ eram do sexo masculino e $10 \%$ do sexo feminino. Além disso, o tipo de anemia mais frequente, classificada de acordo com a avaliação dos índices hematiméricos, foi a anemia normocítica e normocrômica, com 17,50\%, enquanto que a anemia microcítica e hipocrômica apresentou incidência de $7,50 \%$. Além disso, houve prevalência de anisocitose em $37,50 \%$ dos participantes.

A partir da avaliação dos dados referidos no presente estudo, pode-se concluir que, de acordo com a amostragem avaliada, a prevalência de anemias na população idosa ainda é preocupante, já que um quarto destes se encontrava com quadros anêmicos.

Como limitações, cita-se o tamanho da amostra e o fato de o estudo ter sido realizado em apenas um município, com características específicas, o que não permite a generalização dos resultados. Assim, sugere-se o desenvolvimento de novos estudos envolvendo amostras maiores e de outras regiões do país para que os resultados possam ser mais abrangentes.

Dessa forma, espera-se, com os dados obtidos na pesquisa, contribuir com a ciência, bem como mostrar a importância do diagnóstico e a necessidade de se implantar programas de promoção à saúde do idoso, com foco no diagnóstico e tratamento de anemias neste grupo populacional. 


\section{REFERÊNCIAS BIBLIOGRÁFICAS}

BONJORNO, Andressa Francielli, et al. Prevalência de hematócritos alterados na população idosa do município de Araraquara participante da XXI Semana de Assistência Farmacêutica Estudantil (SAFE). Rev. Ciên Farm Básica, Apl., Araraquara, v. 40 Supl. 1, Setembro 2019.

BRASIL, Ministério da Saúde. Secretaria de Atenção à Saúde. Departamento de Atenção Basica. NutriSUS: Guia de evidências: Estratégia de fortificação da alimentação infantil com micronutrientes (vitaminas e minerais) em pó / Ministério da Saúde. Secretaria de Atenção à Saúde. Departamento de Atenção Básica. Brasília: Ministério da Saúde 2015. 50 p.: il.

BRUM, Mabel Figueiredo. FRIZZO, Matias. Eritrograma: Novas perspectivas de análise. 2013. 14f. Monografia (Pós-graduação em Hematologia Laboratorial) - Universidade Regional do Noroeste do Estado do Rio Grande do Sul (UNIJUI), IJUÍ.

BUFFON, et al. Prevalência e caracterização da anemia em idosos atendidos pela Estratégia Saúde da Família. Rev. bras. geriatr. Gerontol, vol.18, n.2, pp.373-384, 2015.

BUFFON, P. L. D. Estudo da prevalência de anemia em idosos atendidos pela Estratégia Saúde da Família do município de Porto Alegre. 2014. 80 f. Dissertação (Mestrado em Gerentologia Biomédica) - Universidade Católica de Rio Grande do Sul, Porto Alegre.

CORONA, L. P.; DUARTE, Y. A. D.; LEBRÃO, M. L. Prevalência de anemia e fatores associados em idosos: evidências do Estudo SABE. Revista de Saúde Pública, v. 48, n. 5, p. 723 - 731. São Paulo, 2014.

CRISTO, et al. Avaliação do perfil hematológico da linhagem vermelha em idosos atendidos no Laboratório de Análises Clínicas (LAC) da Faculdade de Ciências Farmacêuticas da Universidade Federal do Pará. Anais do III Congresso de Educação em Saúde da Amazônia (COESA), Universidade Federal do Pará - 12 a 14 de novembro de 2014.

GUALANDRO, S. F. M.; HOJAIJ, N. H. S.; FILHO, W. J. Deficiência de ferro no idoso. Revista brasileira de hematologia e hemoterapia, vol.32, suppl.2, pp.57-61, São Paulo, 2010.

INSTITUTO BRASILEIRO DE GEOGRAFIA E ESTATÍSTICA. Cidades e Estados. Brejo Santo. Disponível em: <https://www.ibge.gov.br/cidades-e-estados/ce/brejo-santo.html.

LOPES, R. et al. Prevalência de anemia em idosos internados em enfermarias gerais de um hospital universitário. Revista Brasileira de Ciências do Envelhecimento Humano, v. 12, n. 1, p. 25-34, 2016.

MACHADO, Ísis Eloah; MALTA, Deborah Carvalho; BACAL, Nydia Strachman; ROSENFLED, Luiz Gastão Mange. Prevalência de anemia em adultos e idosos brasileiros. Rev bras epidemiol, 22, sulp 2, 2019.

MATOS, J. F.; DUSSE, L. M. S.; GOMES, K. B.; STUBERT, R. V. B.; FERREIRA, M. F. R.; MOREIRA, R. C. N.; FERNANDES, A. P. S. M.; FARIA, J. R.; CARVALHO, M. G. O hemograma nas anemias microcíticas e hipocrômicas: aspectos diferenciais. Rev. J. Bras. Patol. Med. Lab. São Paulo, v. 48, n. 4, p. 255 - 258, 2012.

MILAGRES, C. S.; FRANCESCHINI, S. C. C.; PRIORE, S. E.; LIMA, L. M.; RIBEIRO, A. Q. Prevalência e Etiologia da anemia em idosos: uma revisão integral. Medicina (Ribeirão Preto Online), v. 48 n. 1, p. 99 - 107, 2014.

NEKEL, J. C. Anemia carencial em idosos por deficiência de ferro e ácido fólico e 
vitamina B12. 2013. 23 f. Monografia (Pós Graduação em Hematologia Laboratorial) Universidade Regional do Noroeste do Estado do Rio Grande do Sul (UNIJUI), IJUÍ.

ROSENFELD, Ricardo. Hemograma. Bras. Patol. Med. Lab. Volume 48. N. 4, Agosto 2012.

SANTOS, I. S. Prevalência de anemia em idosos, causas de persistência ou recorrência e sua relação com demência: resultados do São Paulo Ageing and Health Study. 2009. Tese (Doutorado em Emergências Clínicas) - Faculdade de Medicina, Universidade de São Paulo, São Paulo, 2009. Disponível em: <http://www.teses.usp.br/teses/disponiveis/5/5159/tde05022010-164250/>. Acesso em: 16-03-2016.

SOUSA, Natane Daiana Silva. Prevalência de anemia e fatores associados em idosos. 2015. 68 f. Dissertação (Mestrado em Saúde Pública) - Universidade Estadual da Paraíba, Campina Grande.

TOMIYA, M. T. O.; PINHO, C. P. S.; CABRAL, P. C. Anemia e fatores associados: um estudo em idosos hospitalizados. Rev Bras Nutr Clin, v. 29, n. 4, p. 296-303, 2014. 